\title{
Low Frequencies of Physical Activity in Patients with Spinal Cord Injury
}

\author{
Cristiany Lopes Munhoz ${ }^{1}$, Thais Massetti ${ }^{2 *}$, Karina Fontes Csibak ${ }^{1}$, Lilian Del Ciello de \\ Menezes $^{2}$, Talita Dias da Silva ${ }^{3}$, Claudia Arab ${ }^{3}$, Amanda Vitória Lacerda de Araújo ${ }^{5}$, Carlos \\ Bandeira de Mello Monteiro ${ }^{2,5}$, Bruna Eriko Matsuda Marangoni ${ }^{4}$ \\ ${ }^{I}$ Federal University of São Paulo - Paulista School of Medicine - São Paulo, SP, Brazil \\ ${ }^{2}$ Post-graduate Program in Rehabilitation Sciences - Faculty of Medicine - University of São Paulo, São Paulo, \\ SP, Brazil \\ ${ }^{3}$ Post-graduate Program in Cardiology - Federal University of São Paulo - Paulista School of Medicine - São \\ Paulo, SP, Brazil \\ ${ }^{4}$ Irmandade da Santa Casa de Misericórdia de São Paulo - ISCMSP - São Paulo, SP, Brazil \\ ${ }^{5}$ University of São Paulo, School of Arts, Sciences and Humanities - EACH - USP, São Paulo, Brazil
}

*Corresponding Author: Thais Massetti, Post-graduate Program in Rehabilitation Sciences - Faculty of Medicine - University of São Paulo, São Paulo, SP, Brazil, Email: thaismassetti@gmail.com

\begin{abstract}
The amount of disability associated with a spinal cord injury (SCI) is unique to each injury and depends on both the level and completeness of damage. However, physical deconditioning is a common consequence of SCI and can further exacerbate the impact of injury, leading to an increased risk of chronic secondary health complications, such as cardiovascular disease, which are very common causes of death in people with chronic SCI. This study aimed to verify the effects of a physical activity program once or twice a week on cardiorespiratory parameters and physical conditioning in individuals with SCI. Eleven participants were divided into two groups: physical therapy once a week (group 1) or twice a week (group 2). The evaluation of physical conditioning and cardiorespiratory parameters (i.e. heart rate, blood pressure, respiratory rate) was used the adapted 6-minute walk test (6MWT). The results showed no statistical difference in cardiorespiratory parameters. A marginal significance in time to finish the adapted 6MWT and statistical significance in heart rate were observed in group 1, but not in group 2. We conclude that low frequencies of PA, both once or twice a week, could not change the cardiovascular behavior, supporting higher frequencies of training for this goal.
\end{abstract}

Keywords: "spinal cord injury", "physical activity", "physical therapy"

\section{INTRODUCTION}

Spinal Cord Injury (SCI) is a traumatic event characterised by disturbances of sensory, motor or autonomic functions, with an influence on the physical, psychological and social well-being of individuals ${ }^{1}$. Thus, SCI is associated with high levels of disability ${ }^{2}$ and functional incapacity ${ }^{3}$.

The disability associated with SCI is unique to each injury and is related to both the level and completeness of spinal cord damage. After SCI, the loss of muscle mass due to denervation and inactivity and a concomitant decrease in strength ${ }^{4}$. In addition, SCI can modify the autonomic nervous system, local blood flow, peripheral circulation, cardiac output, demand for oxygen to the muscle, and blood flow to the lower limbs ${ }^{5-8}$.
These factors impact on physical activity (PA) level and physiological responses, resulting in an increased sedentary lifestyle, physical deconditioning and fatigue 9-11. Therefore, physical deconditioning and SCI alterations can exacerbate the impact of the injury, increasing risks for chronic secondary health complications 12, 13. Cardiovascular and circulatory diseases negatively affect the quality of life of SCI individuals ${ }^{3}$, and are the most common causes of death in this population ${ }^{14,15}$.

In cases of incomplete SCI, the CNS is capable of plastic change, and the rehabilitation (i.e. PA) must compensate the impairment to maximise the potential for recovery and reduce risks for secondary complications ${ }^{15}, 16$. The PA in 
programs of rehabilitation show positive effects on cardiovascular parameters ${ }^{17}$, motor and sensory functions ${ }^{18}$, psychosocial factors ${ }^{19}$, well-being, functionality ${ }^{20}$, quality of life and everyday activities ${ }^{11}$. The appropriate prescription of exercise programs is needed to improve health status and overall quality of life ${ }^{3}$. In the review study by Ginis et al. (2011) ${ }^{12}$, the minimum frequency required to produce improvements in physical capacity and muscular strength of SCI individuals was 2 days/w or $\geq 3$ days/w with high or moderate intensity.

Jacobs et al. (2002) ${ }^{22}$ applied a protocol of 16 weeks, ( $3 \mathrm{~d} / \mathrm{w})$, including mobility activities, strength, coordination and aerobic resistance in 13 individuals with SCI. After this protocol, the physical conditioning and maximum resistance showed significant improvement. Cardoso et al. (2003) ${ }^{10}$ investigated 9 months of twice-weekly strength and endurance training in SCI patients, with significant increases on strength, arm ergo meter performance and quality of life of the participants. Another study indicated an improvement of aerobic activity and strength in individuals with SCI engaged in at least 20 minutes of moderate to vigorous intensity aerobic activity twice a week ${ }^{14}$.

These studies showed a positive impact on the PA and function of most SCI individuals. However, due to several factors (financial, distance from clinic, time free) the patients are only available for PA once a week. However, there is a lack of empirical evidence regarding this frequency of training. Ramadi et al. (2016) ${ }^{23}$ evaluated people with coronary artery disease comparing PA training once and twice a week and found that both frequencies of training were not enough to change cardiopulmonary parameters.

Thus, this study aimed to verify the effects of a physical activity program once and twice a week on cardiorespiratory parameters and physical conditioning in individuals with SCI. Our hypothesis is that the frequency of PA is directly proportional to cardiorespiratory benefits and physical conditioning in individuals with SCI.

\section{METHOD}

A prospective study with subjects with SCI was developed in the Neurological Adult Physical Therapy Sector of Santa Casa of Misericordia of Sao Paulo (ISCMSP) after approval of the Ethics Committee of the same institution was gained, with project number 31609.
Eleven subjects with chronic SCI (ten males and one female) were included in this study. All participants agreed to participate and provided written informed consented indicating voluntary participation. Eligibility requirements included subjects with permanent incomplete SCI (paraplegic and tetraplegic). Exclusion criteria were: cardiovascular problems, resting blood pressure (BP) above 140/90mmHg, complications in the upper limbs (UL) that prevent the exercises practice (e.g. fractures or pain), physically active patients (i.e. patients that performed any PA or sports), not consenting to participate, and frequency less than $70 \%$ of the total PA program.

The participants were divided into two groups which patients were assigned according to their availability to attend the PA program. The participants were allocated to Group $1(\mathrm{n}=5)$, who performed PA once a week, and Group 2 (n $=6$ ), undergoing PA twice a week.

To assess physical conditioning, we adapted the 6-min walk test (6MWT). The adapted walk test requires travelling 800 metres in the wheelchair without interruption, within the maximum heart rate (MHR), and has been adapted for this distance due to the lack of a suitable place in which to accomplish the same 10 . The result of the adapted 6MWT is the time spent to complete the course.

The evaluated cardiorespiratory parameters were heart rate (HR) - Oregon Scientific (Heart Rate Monitor model: HR102), blood pressure (BP) - sphygmomanometers and stethoscope $\mathrm{HB}$, and respiratory rate (RR). These parameters were evaluated before and after the PA program, beyond the perimeter of the upper limbs $(5,10$ and $15 \mathrm{~cm}$ with fixed point in the fold).

The 12-week PA program was composed of functional exercises to strengthen the muscle groups used the most to ride in a wheelchair, which are the elbow and shoulder flexors and extensors, and shoulder abductors and adductors 24. Our protocol followed the guidelines proposed by Lalor et al. (2005) and Ginis et al. (2011) 12, 14. The PA program sessions occurred once (Group 1) or twice a week (Group 2), and consisted of the following exercises: (i) rolling for both sides during 5 minutes; (ii) 30 repetitions of bending pectoral work and bending triceps work; (iii) cat stance for 3 minutes for trunk control; (iv) decubitus ventral without support of the arms and with symmetric hands for 5 minutes; (v) the same exercise with alternating hands for 5 minutes; 
and finally (vi) dragged into the sitting position for 5 minutes to strengthen upper limbs and assess trunk control.

\section{DATA ANALYSIS}

Statistical analyses were conducted using SPSS software (version 20.0) to calculate averages and standard deviations. The data are expressed as the mean \pm standard deviation (SD) of the measurements. Kolmogorov-Smirnov was conducted to verify data distribution, and Levine's test to assess variances in homogeneity. ANOVA with repeated measures was used to compare the participants before and after the PA program. The statistical significance level was set at $\alpha=0.05$.

\section{Results}

Table 1 shows the clinical characteristics of each of the 11 participants. Group 1 (Intervention once a week) included five subjects age $37 \pm 14$ years, while group 2 (intervention twice a week) included six individuals aged $35 \pm 12$ years.

After 12 weeks of PA program, no significant differences were found in cardiorespiratory parameters for both groups ( $\mathrm{P}>0.05$, Table 2$)$. A marginal significance on HR 1 minute postadapted 6MWT was observed in group 1 . The significant effect of a PA program in decreased time to finish adapted 6MWT in group 1.

Table1. Characterization of the sample groups

\begin{tabular}{|l|c|c|c|}
\hline & Subjects & Age (years) & Level of Injury \\
\hline \multirow{4}{*}{ Group 1 (Once a week) } & $\mathbf{1}$ & 57 & C7/L3 \\
\cline { 2 - 4 } & $\mathbf{2}$ & 28 & T12 \\
\cline { 2 - 4 } & $\mathbf{3}$ & 21 & $\mathrm{~T} 10$ \\
\cline { 2 - 4 } & $\mathbf{4}$ & 36 & $\mathrm{~T} 6$ \\
\cline { 2 - 4 } & $\mathbf{5}$ & 42 & $\mathrm{~T} 12$ \\
\hline \multirow{3}{*}{ Group 2 (Twice a week) } & $\mathbf{1}$ & 24 & $\mathrm{~T} 10$ \\
\cline { 2 - 4 } & $\mathbf{2}$ & 33 & $\mathrm{~T} 10$ \\
\cline { 2 - 4 } & $\mathbf{3}$ & 25 & $\mathrm{C} 5$ \\
\cline { 2 - 4 } & $\mathbf{4}$ & 33 & $\mathrm{~T} 10$ \\
\cline { 2 - 4 } & $\mathbf{5}$ & 40 & $\mathrm{~T} 11$ \\
\cline { 2 - 4 } & $\mathbf{6}$ & 57 & $\mathrm{C} 5 / \mathrm{C} 6$ \\
\hline
\end{tabular}

Table2. Description of the physiological variables and time to finish the 6MWT (adapted)

\begin{tabular}{|c|c|c|c|c|c|c|}
\hline & \multicolumn{3}{|c|}{ Once a week } & \multicolumn{3}{|c|}{ Twice a week } \\
\hline & Pre treatment & Post treatment & $\boldsymbol{P}$ & $\begin{array}{c}\text { Pre } \\
\text { treatment }\end{array}$ & Post treatment & $p$ \\
\hline SBP (mmHg) & $126 \pm 11.4$ & $130 \pm 7.1$ & 0.317 & $110 \pm 23.7$ & $111.7 \pm 21.4$ & 0.783 \\
\hline DBP, (mmHg) & $80 \pm 7.1$ & $90 \pm 0.0$ & 0.102 & $70 \pm 14.1$ & $73.3 \pm 12.1$ & 0.564 \\
\hline$\Delta$ HR, (bpm) & $53.8 \pm 19.6$ & $63.8 \pm 16.9$ & 0.498 & $71 \pm 21.0$ & $51.3 \pm 29.9$ & 0.116 \\
\hline$\Delta \mathrm{RR},(\mathrm{rpm})$ & $9.80 \pm 3.5$ & $12.2 \pm 5$ & 0.416 & $10.7 \pm 4.4$ & $6.8 \pm 6.6$ & 0.249 \\
\hline$\Delta \mathrm{SBP}(\mathrm{mmHg})^{\mathrm{a}}$ & $6.20 \pm 5.2$ & $0.20 \pm 15$ & 0.416 & $10.2 \pm 15.5$ & $23.5 \pm 25.8$ & 0.518 \\
\hline$\Delta \mathrm{DBP},(\mathrm{mmHg})^{\mathrm{a}}$ & $-14 \pm 32.1$ & $-6 \pm 8.9$ & 1 & $11.7 \pm 24.8$ & $0.0 \pm 8.9$ & 0.357 \\
\hline$\Delta$ HR, (bpm) ${ }^{\mathrm{a}}$ & $23.6 \pm 8.3$ & $42.2 \pm 12.7$ & $0.043^{*}$ & $31.8 \pm 20.2$ & $14.7 \pm 32.2$ & 0.173 \\
\hline$\Delta \mathrm{RR},(\mathrm{rpm})^{\mathrm{a}}$ & $3.8 \pm 4.1$ & $6.6 \pm 5.7$ & 0.581 & $6.8 \pm 4.1$ & $2.7 \pm 5.7$ & 0.115 \\
\hline Time to finish & 6'32" \pm 1 1'04"' & 6’08'” $\pm 0{ }^{\prime} 48^{\prime \prime}$ & $0.068 * *$ & 9'30'" \pm 5'37'" & 9'33" \pm 5'15" & 0.600 \\
\hline
\end{tabular}

6MWT Six-Minute Walk Test, SBP Systolic Blood Pressure, DBP Diastolic Blood Pressure; HR: Heart Rate, RR Respiratory Rate, mmHg millimetres of mercury, bpm beats per minute; min minute, rpm repetitions per minute. $\triangle$ post 6MWT values minus pre 6MWT (adapted) values. ${ }^{a}$ values obtained 1 minute after 6MWT (adapted) ending. **Statistical significance; *marginal significance.

\section{DISCUSSION}

The benefits of PA program on aerobic capacity, strength, cardiovascular system, and quality of life in individuals with SCI are clear 10, 12, 14, 22,25 . Therefore, this study aimed to verify the influence of low frequencies of PA programs on cardiorespiratory parameters and physical conditioning of individuals with SCI.
Contrary to our hypothesis, no differences were found between groups. SCI individuals that performed PA once or twice a week presented no significant results on cardiorespiratory parameters. We observed no statistical difference in the values of heart rate, blood pressure (i.e. systolic and diastolic) and respiratory rate in either group, just a statistical 
significance for HR one minute post-adapted $6 \mathrm{MWT}$ in group 1 . In the same group, a marginal significance was observed in time to finish the adapted 6MWT. Our PA program involved functional exercises (i.e. strength exercises for muscles used in riding wheelchair) that have been reported in SCI with associated increases in muscle strength, resistance and functional capacity 26-28.

Duran et al. (2001) 29 also used mobility activities, aerobic resistance, strength, coordination, exercise in water and combined activities. The physical conditioning of SCI was improved. The systematic review of Hicks et al. 30 showed that arm ergometry and wheelchair exercise increase the aerobic capacity 15,17 and muscle strength 15. A combination of resistance plus arm ergometry training improves physical conditioning in SCI. Ginis et al. (2011) 12 showed the same in a systematic review, where the traditional resistance training, arm ergometry and combined aerobic plus resistance exercise indicated improved physical capacity and strength .

We believe that the fact that our program did not involve aerobic training and used sessions of approximately 30 minutes could have influenced the results. After all, short-term aerobic arm exercise performed for patients with SCI improves their exercise capabilities, however these patients require rehabilitation and longterm programs to receive and benefit from aerobic training 31 .However, our results corroborate the findings of Ramadi et al. (2016) 23 , which found no difference between training once or twice a week considering cardiopulmonary parameters in people with coronary artery disease, even with aerobic training of approximately 60 minutes.

In the study of Davis et al. (1987) 32, a short period of arm training was insufficient to increase cardiac function in paraplegic subjects, on this account, it is likely that although the cardiac parasympathetic flow withdrawal response is preserved in quadriplegic individuals, the rapid acceleration of HR at the beginning of exercise and the rapid deceleration after exercise 33. However, some improvement was observed on pre-loading due to increased venous tone and more effective operation of the muscle pump after training. Milia et al. (2014)34 examined haemodynamic response to muscle activation in SCI with a one-year training period. They found that mean blood pressure response was significantly increased after the period of training, enhancement of HR, cardiac output, ventricular filling rate, and end diastolic volume responses. The authors highlighted that parameters of the exercise program are predictors for beneficial cardiovascular changes.

In short, the priority for rehabilitation in patients with spinal cord injury is the early onset of physical activity, since It optimize recovery and decrease or prevent the degree of post-ICS deconditioning that occurs after weeks of rest and can affect the final functional capacity of an individual 35 .

Some limitations of this study must be regarded. The small sample size does not allow our results to be generalised to the entire population with SCI. The measurements used may not be sufficient to detect cardiorespiratory changes. Future studies including a larger sample size should be conducted to clarify the cardiovascular effects of exercise performed once or twice a week.

\section{CONCLUSiON}

Our findings showed that low frequencies of PA, either once or twice a week, could not change the cardiovascular behaviour, supporting higher frequencies of training for this goal.

\section{REFERENCES}

[1] Yang H, Liu C-c, Wang C-Y, et al. Therapeutical Strategies for Spinal Cord Injury and a Promising Autologous Astrocyte-Based Therapy Using Efficient Reprogramming Techniques. Molecular neurobiology. 2015:117.

[2] Guilcher SJ, Casciaro T, Lemieux-Charles L, Craven C, McColl MA, Jaglal SB. Social networks and secondary health conditions: the critical secondary team for individuals with spinal cord injury. The journal of spinal cord medicine. 2012;35(5):330-342.

[3] Barbeau EB, Meilleur AA, Zeffiro TA, Mottron L. Comparing Motor Skills in Autism Spectrum Individuals With and Without Speech Delay. Autism Res. Mar 292015.

[4] Martin Ginis KA, Jorgensen S, Stapleton J. Exercise and sport for persons with spinal cord injury. Pm r. Nov 2012;4(11):894-900.

[5] Jain NB, Ayers GD, Peterson EN, et al. Traumatic spinal cord injury in the United States, 1993-2012. Jama. Jun 9 2015;313(22):2236-2243.

[6] Yang H, Liu CC, Wang CY, et al. Therapeutical Strategies for Spinal Cord Injury 
and a Promising Autologous Astrocyte-Based Therapy Using Efficient Reprogramming Techniques. Mol Neurobiol. Apr 122015.

[7] Zaniz FL, de Lima E, Júnior EVP, Frota PB, Gonçalves CBH, Moraes MR. Análise do duplo produto no treinamento de força em séries com características metabólicas e tensionais. Revista Brasileira de Prescrição e Fisiologia do Exercício (RBPFEX). 2008;2(7):5.

[8] Morawietz C, Moffat F. Effects of locomotor training after incomplete spinal cord injury: a systematic review. Arch Phys Med Rehabil. Nov 2013;94(11):2297-2308.

[9] do Nascimento LG, da Silva SML. Benefícios da atividade física sobre o sistema cardiorrespiratório, como também, na qualidade de vida de portadores de lesão medular: uma revisão. RBPFEX-Revista Brasileira de Prescrição e Fisiologia do Exercício. 2011;1(3).

[10] Cardoso JR, Erichsen OA, Nampo FK, Tookuni KS, Dourado VZ. Condicionamento aeróbico em indivíduos portadores de lesão medular. Fisioter. Mov. 2003;16(1):25-28.

[11] Haisma JA, van der Woude LH, Stam HJ, Bergen MP, Sluis TA, Bussmann JB. Physical capacity in wheelchair-dependent persons with a spinal cord injury: a critical review of the literature. Spinal Cord. Nov 2006;44(11):642652.

[12] Ginis KA, Hicks AL, Latimer AE, et al. The development of evidence-informed physical activity guidelines for adults with spinal cord injury. Spinal Cord. Nov 2011;49(11):10881096.

[13] Wirz M, van Hedel HJ, Rupp R, Curt A, Dietz $\mathrm{V}$. Muscle force and gait performance: relationships after spinal cord injury. Arch Phys Med Rehabil. Sep 2006;87(9):1218-1222.

[14] Lalor EC, Kelly SP, Finucane C, et al. Steadystate VEP-based brain-computer interface control in an immersive 3D gaming environment. EURASIP journal on applied signal processing. 2005;2005:3156-3164.

[15] Nakafuji A, Tsuji K. Learning and transfer in two perceptual-motor skills in Duchenne muscular dystrophy. Percept Mot Skills. Oct 2001;93(2):339-352.

[16] Heller KW, Mezei PJ, Avant MJT. Meeting the Assistive Technology Needs of Students with Duchenne Muscular Dystrophy. Journal of Special Education Technology. 2009;23(4):2008-2009.

[17] Hoekstra F, van Nunen MP, Gerrits KH, Stolwijk-Swuste JM, Crins MH, Janssen TW. Effect of robotic gait training on cardiorespiratory system in incomplete spinal cord injury. J Rehabil Res Dev. 2013; 50(10): 1411-1422.
[18] Macklin R, Brooke V, Calabro F, Ellaway P, Perez MA. Discrepancies between clinical assessments of sensory function and electrical perceptual thresholds after incomplete chronic cervical spinal cord injury. Spinal Cord. 2016;54(1):16-23.

[19] Martin Ginis KA, Papathomas A, Perrier MJ, Smith B. Psychosocial factors associated with physical activity in ambulatory and manual wheelchair users with spinal cord injury: a mixed-methods study. Disabil Rehabil. Jun 24 2015:1-6.

[20] Ho CH, Triolo RJ, Elias AL, et al. Functional electrical stimulation and spinal cord injury. Phys Med Rehabil Clin N Am. Aug 2014;25(3):631-654, ix.

[21] Brodwin MG, Star T, Cardoso E. Computer assistive technology for people who have disabilities: Computer adaptations and modifications. Journal of rehabilitation. 2004; 70(3):28.

[22] Jacobs PL, Mahoney ET, Nash MS, Green BA. Circuit resistance training in persons with complete paraplegia. J Rehabil Res Dev. JanFeb 2002;39(1):21-28.

[23] Ramadi A, Buijs DM, Threlfall TG, et al. Long-term Physical Activity Behavior After Completion of Traditional Versus Fast-track Cardiac Rehabilitation. J Cardiovasc Nurs. Apr 222016.

[24] Janssen TW, Dallmeijer AJ, Veeger DJ, van der Woude LH. Normative values and determinants of physical capacity in individuals with spinal cord injury. J Rehabil Res Dev. Jan-Feb 2002;39(1):29-39.

[25] Greve J, Casalis MEP, Barros Filho TEPd. Diagnóstico e tratamento da lesão da medula espinal. São Paulo: Roca. 2001;15.

[26] Munce SE, Straus SE, Fehlings MG, et al. Impact of psychological characteristics in selfmanagement in individuals with traumatic spinal cord injury. Spinal Cord. Jun 92015.

[27] Elliott TR, Kennedy P. Treatment of depression following spinal cord injury: an evidence-based review. Rehabilitation Psychology. 2004; 49(2):134.

[28] Smith E, Brosnan M, Comiskey C, Synnott K. Road collisions as a cause of traumatic spinal cord injury in ireland, 2001-2010. Top Spinal Cord Inj Rehabil. Spring 2014;20(2):158-165.

[29] Duran FS, Lugo L, Ramirez L, Eusse E. Effects of an exercise program on the rehabilitation of patients with spinal cord injury. Arch Phys Med Rehabil. Oct 2001;82(10):1349-1354.

[30] Hicks A, Ginis KM, Pelletier C, Ditor D, Foulon B, Wolfe D. The effects of exercise training on physical capacity, strength, body composition and functional performance among 
adults with spinal cord injury: a systematic review. Spinal cord. 2011;49(11):1103-1127.

[31] Akkurt H, Uzumcugil Karapolat H, Kirazli Y, Kose T. The effects of upper extremity aerobic exercise on exercise capacity and other health issues in patients with spinal cord injury: a randomized controlled study. Eur J Phys Rehabil Med. Nov 082016.

[32] Davis GM, Shephard RJ, Leenen FH. Cardiac effects of short term arm crank training in paraplegics: echocardiographic evidence. Eur J Appl Physiol Occup Physiol. 1987;56(1):90-96.

[33] Takahashi M, Matsukawa K, Nakamoto T, et al. Control of heart rate variability by cardiac parasympathetic nerve activity during voluntary static exercise in humans with tetraplegia. J Appl Physiol (1985). Nov 2007;103(5):16691677.

[34] Milia R, Roberto S, Marongiu E, et al. Improvement in hemodynamic responses to metaboreflex activation after one year of training in spinal cord injured humans. Biomed Res Int. 2014;2014:893468.

[35] Scivoletto G, Morganti B, Molinari M. Early versus delayed inpatient spinal cord injury rehabilitation: an Italian study. Arch Phys Med Rehabil. Mar 2005;86(3):512-516.

Citation: Cristiany Lopes Munhoz, Thais Massetti, Karina Fontes Csibak, Lilian Del Ciello de Menezes, Talita Dias da Silva, Claudia Arab et al. Low Frequencies of Physical Activity in Patients with Spinal Cord Injury. ARC Journal of Neuroscience. 2017; 2(3):7-12. doi:dx.doi.org/10.20431/2456-057X.0203003.

Copyright: (C) 2017 Authors. This is an open-access article distributed under the terms of the Creative Commons Attribution License, which permits unrestricted use, distribution, and reproduction in any medium, provided the original author and source are credited. 